\title{
Theoretical and Experimental Investigation of Effects of Toroidal Field Ripple on Poloidal Beta, Internal Inductance and Shafranov Shift in IR-T1 Tokamak
}

\author{
Ahmad Salar Elahi*, Mahmood Ghoranneviss \\ Plasma Phy sics Research Center, Science and Research Branch, Islamic Azad University, Tehran, Iran
}

\begin{abstract}
In this paper we presented an investigation of effects of the toroidal field ripple (TF ripp le) (due to fin ite nu mber of the toroidal field coils) on the poloidal beta, internal inductance, and Shafranov shift in IR-T1 Tokamak. For these purposes, array of magnetic probes and also a diamagnetic loop with its compensation coil were designed, constructed and installed on outer surface of the IR-T 1. A mplitude of the TF ripple is obtained 0.01, and also the effects of TF ripple on the poloidal beta, internal inductance, and Shafranov shift were discussed. In the high field side region of toka mak chamber, the TF ripple effects is the decreasing and increasing of the poloidal beta and internal inductance, respectively, whereas the low field side have inverse situations. Also no sensible variation observed in the Shafranov shift due to TF ripple on IR-T1 plasma.
\end{abstract}

Keywords Tokamak, Toroidal Field Ripple, Poloidal Beta, Internal Inductance, Shafranov Shift

\section{Introduction}

In most of toro idal plasma equilibrium studies, tokamak equilibria are investigated as axisymmetric systems (two-dimensional systems). Although this symmetry offers many advantages for its analys is, but realistic tokamaks consists of fin ite number of toroidal field coils (TF). Then, this discreteness yields the toroidal field ripples (a periodic variation of the toroidal magnetic field) (TF ripples). In other words, realistic tokamaks could not be axis ymmetric configurations. Most of the TF ripple studies have been done on effects of the TF ripple on confinement of the high energy alpha particles, formation of internal transport barriers, plasma rotation, and $\mathrm{H}$-mode performance. In IR-T 1 Tokamak, which it is a small, low beta and large aspect ratio tokamak with a circular cross section (see Table 1), the number $\mathrm{N}$ of TF coils is 16 , and then the period of the TF ripple was $22.5^{\circ}$. In this paper we presented the effects of the TF ripple on determinations of the poloidal beta, plas ma internal inductance, and therefore the Shafranov parameter and Shafranov shift in IR-T1. Determinations of the poloidal beta, internal inductance, and Shafranov shift are essential for tokamak experiments and optimized operation. Also some of the plasma informations can be deduced from these

* Corresponding author:

salari_phy@yahoo.com (Ahmad Salar Elahi)

Published online at http://journal.sapub.org/jnpp

Copyright (C) 2012 Scientific \& Academic Publishing. All Rights Reserved parameters, such as plasma toroidal current profile, plas ma energy, plasma energy confinement time, and magnetohydrodynamics (MHD) instabilities.

Magnetic diagnostics, in particular diamagnetic loop are commonly used in tokamaks to measure the variation of toroidal flux induced by the plasma and then the poloidal Beta. On the other hand, the magnetic fields distribution outside the plasma provides the measurement of the combination of poloidal beta and internal inductance, via the Shafranov parameter. Then measurement of the Shafranov parameter from the magnetic probes and poloidal beta from the diamagnetic loop gives a value of the internal inductance. Also Shafranov shift can be determined from the Shafranov parameter. Although the questionable parameters in this work usually analy zed as a global plasma parameters, but we present theoretical and experimental investigation of the TF ripple on the estimate of these parameters from localized measurements. Because of dependence of the toroidal field on the TF ripple amplitude, therefore we expect that these parameters are also depending on TF ripple amplitude[1-51]. Brief approach for determinations of the TF ripple and Shafranov parameter by the discrete magnetic probes will be presented in section 2. Diamagnetic loop method for measurement of the poloidal beta, internal inductance, and then the Shafranov shift will be presented in section 3 . Details of design and construction of magnetic probe and diamagnetic loop will be presented in section 4. Experimental results will be discussed in section 5 . Summary and conclusion will be discussed in section 6 . 
Table 1. Main Parameters of the IR-T1 Tokamak

\begin{tabular}{|c|c|}
\hline Parameters & Value \\
\hline Major Radius & $45 \mathrm{~cm}$ \\
\hline Minor Radius & $12.5 \mathrm{~cm}$ \\
\hline Toroidal Field & $\langle 1.0 \mathrm{~T}$ \\
\hline Plasma Current & $\langle 40 \mathrm{kA}$ \\
\hline Discharge Duration & $\langle 35 \mathrm{~ms}$ \\
\hline Electron Density & $0.7-1.5 \times 10^{13} \mathrm{~cm}^{-3}$ \\
\hline Toroidal Field Coils & 16 \\
\hline
\end{tabular}

\section{Determinations of the TF Ripple and Shafranov parameter by the Discrete Magnetic Probes}

A simple analytic model of the toroidal magnetic field strength widely used in the analysis is the following:

$$
B_{\phi}(\theta, \phi)=B_{0}(1-\varepsilon \cos \theta-\delta \cos \mathrm{N} \phi),
$$

where $B_{0}$ is the toroidal magnetic field at center of the tokamak chamber, $\theta$ and $\phi$ are poloidal and toroidal angles respectively, $\varepsilon$ is the inverse aspect ratio, $N$ is the number of the toroidal field coils, and $\delta$ is the amplitude of the TF ripple where defined as:

$$
\delta=\frac{\delta B}{\langle B\rangle_{\phi}} \approx \frac{B_{\max }-B_{\min }}{B_{\max }+B_{\min }} .
$$

In the IR-T1, the number of TF co ils is 16 , then the period of the TF ripple was $22.5^{\circ}$, and the inverse aspect ratio is 0.278 . Although the amplitude of TF ripple is no constant at the diffe rent poloidal angles, we can define the average value of TF ripple amplitude from the Eq. (1):

$$
\begin{aligned}
\delta= & \frac{1}{4}\left(\frac{B_{\phi}(\theta=0, \phi=\pi / N)-B_{\phi}(\theta=0, \phi=0)}{B_{0}}\right)+ \\
& \frac{1}{4}\left(\frac{B_{\phi}(\theta=\pi, \phi=\pi / N)-B_{\phi}(\theta=\pi, \phi=0)}{B_{0}}\right),
\end{aligned}
$$

where these values of the toroidal magnetic fields can be determined using the magnetic probes at above poloidal and toroidal angles. Our measurements using the magnetic pick-up coils on outer surface of the IR-T 1 tokamak show that the amplitude of TF ripple on the sensor position is 0.01 , which is close to the result of mode ling as shown in Fig. (1) (Fig. (1) is a plot of the Eq. (1) at the edge of IR-T1 tokamak plasma).

Also the Shafranov parameter and therefore Shafranov shift relate to the distribution of magnetic fields around the plasma current. Therefore, those can be written in terms of the tangential and normal components of the magnetic field on the contour $\Gamma$ (see Fig. (2)). Distributions of the tangential and normal magnetic fields can be written in the first order of the inverse aspect ratio as follows, respectively[1-5]:

$$
\begin{gathered}
B_{\theta}=\frac{\mu_{0} I_{p}}{2 \pi \mathrm{b}}-\frac{\mu_{0} I_{p}}{4 \pi R_{0}} \times \\
\left\{\ln \frac{a}{b}+1-\left(\Lambda+\frac{1}{2}\right)\left(\frac{a^{2}}{b^{2}}+1\right)-\frac{2 R_{0} \Delta_{s}}{b^{2}}\right\} \cos \theta \\
B_{\rho}=-\frac{\mu_{0} I_{p}}{4 \pi R_{0}} \times \\
\left\{\ln \frac{a}{b}+\left(\Lambda+\frac{1}{2}\right)\left(\frac{a^{2}}{b^{2}}-1\right)+\frac{2 R_{0} \Delta_{s}}{b^{2}}\right\} \sin \theta
\end{gathered}
$$

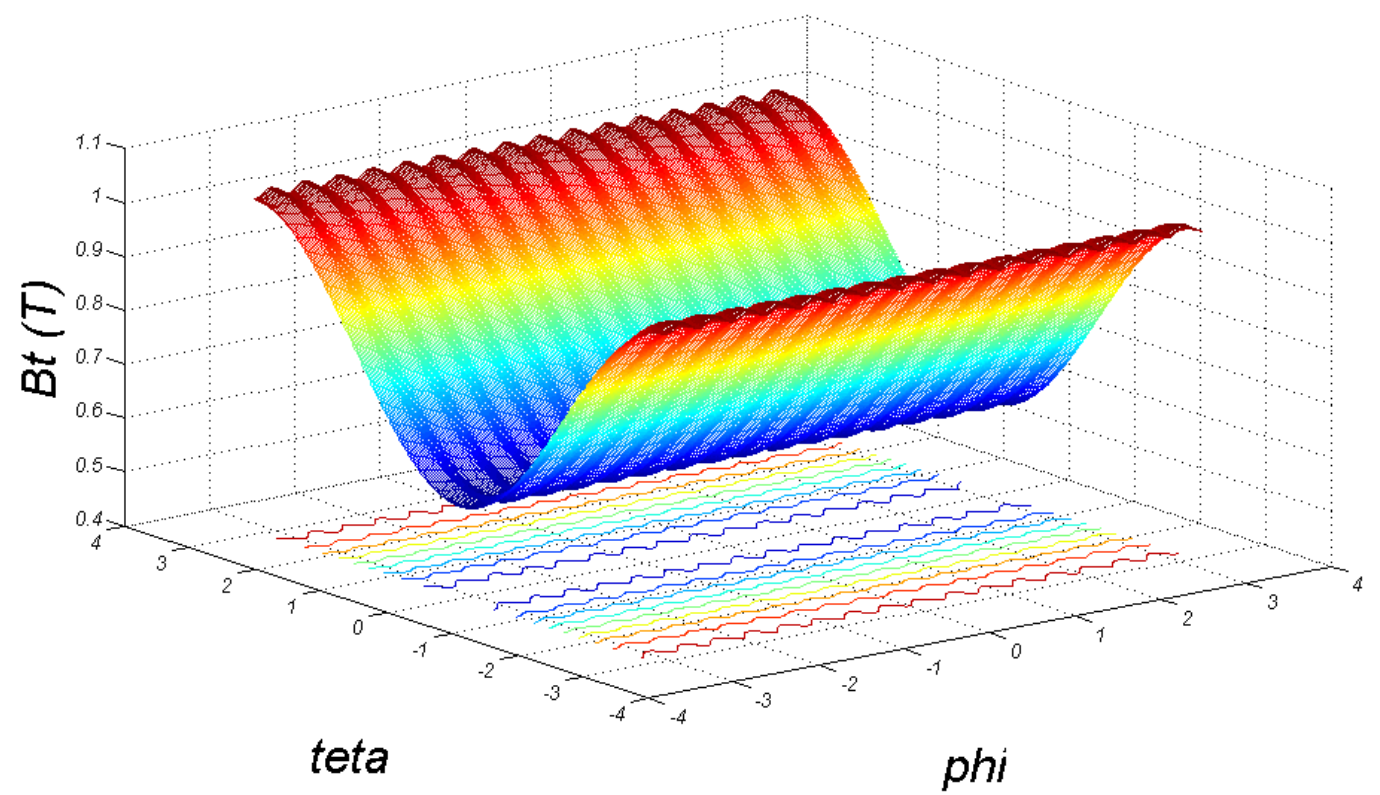

Figure 1. Dependence of the toroidal field to the poloidal and toroidal angles (TF ripple) 
where

$R_{0}$ is the major radius of the vacuumvessel,

$\Delta_{s}$ is the Shafranov shift, $I_{p}$ is the plasma current, $a$ and $b$ are the minor plasma radius and minor chamber radius respectively. These equations accurate for low $\beta$ plasma, large aspect ratio, and circular cross section tokamaks as IR-T 1, and where:

$$
\begin{aligned}
\Lambda= & \beta_{p}+l_{i} / 2-1= \\
& \ln \frac{a}{b}+\frac{\pi R_{0}}{\mu_{0} I_{p}}\left(\left\langle B_{\theta}\right\rangle+\left\langle B_{\rho}\right\rangle\right),
\end{aligned}
$$

where

$$
\begin{aligned}
& \left\langle B_{\theta}\right\rangle=B_{\theta}(\theta=0)-B_{\theta}(\theta=\pi), \\
& \left\langle B_{\rho}\right\rangle=B_{\rho}\left(\theta=\frac{\pi}{2}\right)-B_{\rho}\left(\theta=\frac{3 \pi}{2}\right),
\end{aligned}
$$

and where $\beta_{p}$ is the poloidal beta and $l_{i}$ is the plasma internal inductance. We measured $B_{\theta}$ and $B_{\rho}$ which are the contribution of the plas ma current to the equilib riu m field, after compensating the vacuum field and integrating of the output signals of magnetic probes.

The major approximations in our work are that we suppose the plasma minor radius defined by limiter radius, namely $a$ is constant, and also the TF ripple amplitude at different poloidal angles is constant, whereas the toroidal flux is no constant as a function of phi, and then we have to have field lines which loop back on themselves, in which case we have to have localized poloidal currents. Experimental results will be presented in the section 5 .

\section{Measurements of the Poloidal Beta and Internal Inductance with Diamagnetic Loop}

Diamagnetic loop measures the toroidal diamagnetic flux for the purpose of measurement of the poloidal beta and thermal energy of the plas ma. The toroidal flux produced by the plasma is related to the total perpendicular thermal energy of the plasma. This diamagnetic flux is usually measured with the diamagnetic loop. It is consists of a simple loop that links the plasma column, ideally located in a poloidal direction in order to minimize poloidal field pick-up. Relation between the diamagnetic flux and the poloidal beta is [2-7]:

$$
\beta_{p}=1-\frac{8 \pi B_{\phi}}{\mu_{0}^{2} I_{p}^{2}} \Delta \Phi_{D},
$$

where $\Delta \Phi_{D}=\Phi_{\text {total }}-\Phi_{\text {vacuum }}$ is the diamagnetic flux.

By substituting the Eq. (1) in the Eq. (8) we have:

$$
\beta_{p}=1-\frac{8 \pi B_{0}(1-\varepsilon \cos \theta-\delta \cos \mathrm{N} \phi)}{\mu_{0}^{2} I_{p}^{2}} \Delta \Phi_{D},
$$

where $\Phi_{\text {vacuum }}=\Phi_{T}+\Phi_{O}+\Phi_{V}+\Phi_{E}$, and where $B_{0}$ is the toroidal magnetic field in the absence of the plasma and center of chamber which can be measured using the Ampere law, $I_{p}$ is the plasma current, $\Phi_{T}$ is the toroidal flux because of toroidal field coils, $\Phi_{O}$ and $\Phi_{V}$ are the passing flux through loop due to possible misalignment between ohmic field and vertical field and the diamagnetic loop, and $\Phi_{E}$ is the toroidal field due to eddy current on the vacuum chamber. These fluxes can be compensated with compensation coil (see Fig. (3)) and also using dry runs technique. It must be noted that compensating coil for diamagnetic loop is wrapped out of the plas ma current, and only the toroidal flux (which is induced by the change of toroidal field coil current when plasma discharges) can be received (see Figs. (2),(3)). Therefore, according to above two sections we can find the internal inductance. From Eq. (6) we have:

$$
l i=2\left(\Lambda-\beta_{p}+1\right)
$$

By substituting the Eq. (6) and (9) in Eq. (10), we can write:

$$
\begin{aligned}
l i= & 2 \ln \frac{a}{b}+\frac{2 \pi R_{0}}{\mu_{0} I_{p}}\left(\left\langle B_{\theta}\right\rangle+\left\langle B_{\rho}\right\rangle\right)+ \\
& \frac{16 \pi B_{0}(1-\varepsilon \cos \theta-\delta \cos \mathrm{N} \phi)}{\mu_{0}^{2} I_{p}^{2}} \Delta \Phi_{D},
\end{aligned}
$$

Also, the Shafranov shift is determined from rearranging the Eq. (4):

$$
\begin{aligned}
\Delta_{s}= & \frac{\pi \mathrm{b}^{2}}{\mu_{0} I_{p}}\left\langle B_{\theta}\right\rangle+ \\
& \frac{\mathrm{b}^{2}}{2 \mathrm{R}_{0}}\left[\ln \frac{a}{b}+1-\left(\frac{a^{2}}{b^{2}}+1\right)\left(\beta_{p}+\frac{l_{i}}{2}-\frac{1}{2}\right)\right],
\end{aligned}
$$

where the effects of the TF ripple introduced in the last expression.

Experimental results of effects of the TF ripple on measurements of the poloidal beta, internal inductance, and Shafranov shift, will be presented in the section 5 .

\section{Design and Construction of the Magnetic Probes and Diamagnetic Loop}

In general, a magnetic sensors (magnetic probe or diamagnetic loop) works by Faraday's law and measures component(s) of the local magnetic fields or magnetic flu xes for use in plasma control, equilibrium reconstruction and detection of plasma energy, poloidal beta and MHD instabilities.

In the IR-T 1 tokamak an array of four magnetic probes were designed, two magnetic probes were installed on the circular contour $\Gamma$ of the radius $b=16.5 \mathrm{~cm}$ in angles 
of $\theta=0$ and $\theta=\pi$ to detect the tangential component of the magnetic field $B_{\theta}$, and two magnetic probes are also installed above, $\theta=\pi / 2$, and below, $\theta=3 \pi / 2$, to detect the normal component of the magnetic field $B_{\rho}$, as shown in Fig. (2).

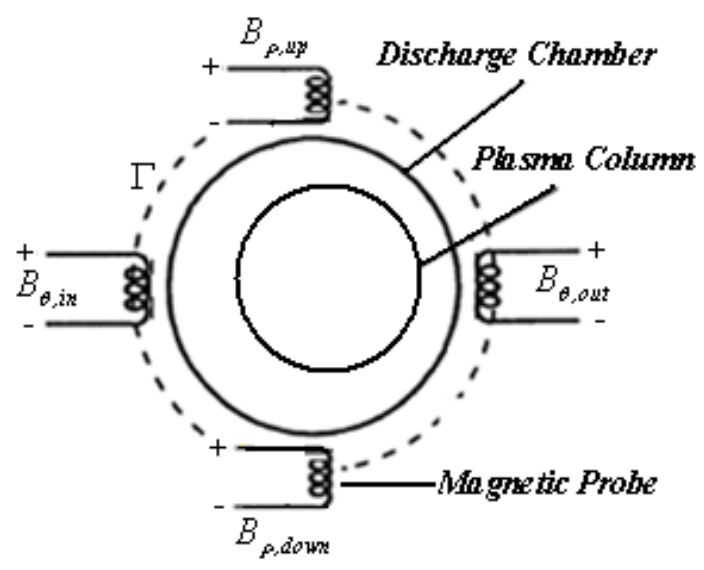

Figure 2. Positions of the four magnetic probes on outer surface of the IR-T1 tokamak chamber

Diamagnetic loop and its compensating coil also were constructed and installed on outer surface of the IR-T1 tokamak, as shown in Fig. (3).

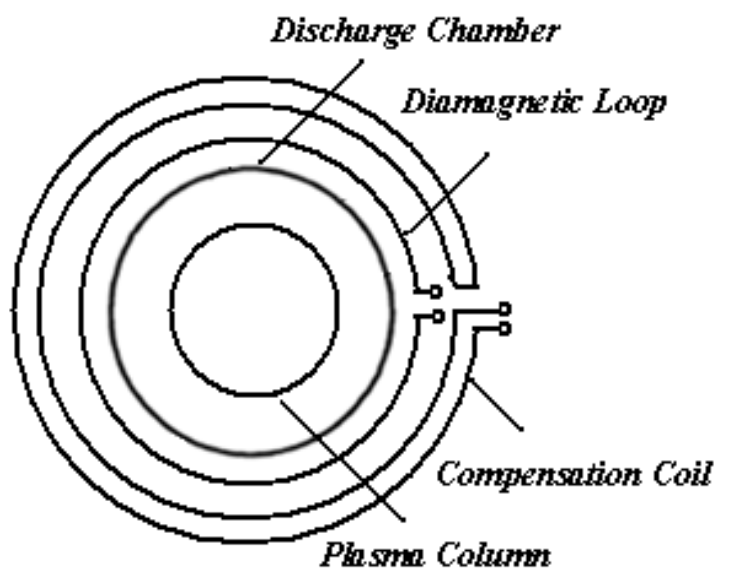

Figure 3. Positions of the diamagnetic loop with its compensation coil on outer surface of the IR-T 1 tokamak chamber

Design parameters of the magnetic pickup coils and diamagnetic loop present in Table 2.

After measurements $\left\langle B_{\theta}\right\rangle$ and $\left\langle B_{\rho}\right\rangle$ from magnetic probes, $\Delta \Phi_{D}$ from diamagnetic loop, $I_{p}$ from Rogowski coil and substituting them in to Eq. (9), Eq. (11), and Eq. (12), the poloidal beta, internal inductance, and Shafranov shift and also the effects of TF ripple on them measured. Experimental results presented in the next section.
Table 2. Design parameters of the magnet ic probe and diamagnetic loop

\begin{tabular}{|c|c|c|}
\hline Parameters & Magnetic Probe & $\begin{array}{c}\text { Diamagnetic } \\
\text { Loop }\end{array}$ \\
\hline $\mathrm{R}$ (Resistivity) & $33 \Omega$ & $100 \Omega$ \\
\hline $\mathrm{L}$ (Inductance) & $1.5 \mathrm{mH}$ & $20 \mathrm{mH}$ \\
\hline $\mathrm{n}$ (Turns) & 500 & 170 \\
\hline $\mathrm{S}$ (Sensitivity) & $0.7 \mathrm{mV} / \mathrm{G}$ & $0.5 \mathrm{~V} / \mathrm{G}$ \\
\hline $\mathrm{f}$ (Frequency Response) & $22 \mathrm{kHz}$ & $5 \mathrm{kHz}$ \\
\hline Effective $n A$ & $0.022 m^{2}$ & $16 m^{2}$ \\
\hline $\mathrm{d}$ (Wire Diameter) & $0.1 \mathrm{~mm}$ & $0.2 \mathrm{~mm}$ \\
\hline$d_{m}$ (Coil Average Radius) & $3 \mathrm{~mm}$ & $175 \mathrm{~mm}$ \\
\hline
\end{tabular}

\section{Experimental Results of the Effects of TF Ripple on the Plasma Parameters}

We used the electric circu it as shown in Figures (4) and (5), for measurements of the magnetic fields and diamagnetic flux, respectively:

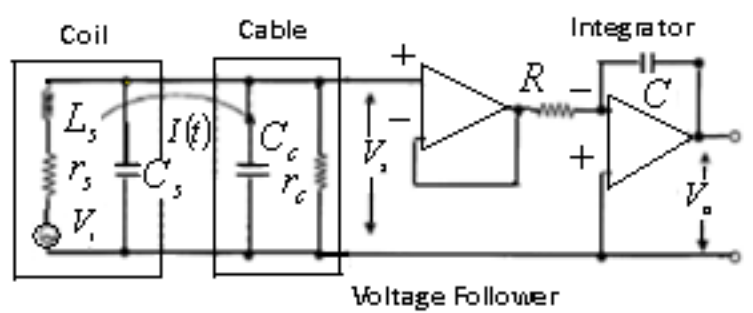

Figure 4. Electric circuit used for the magnetic probes

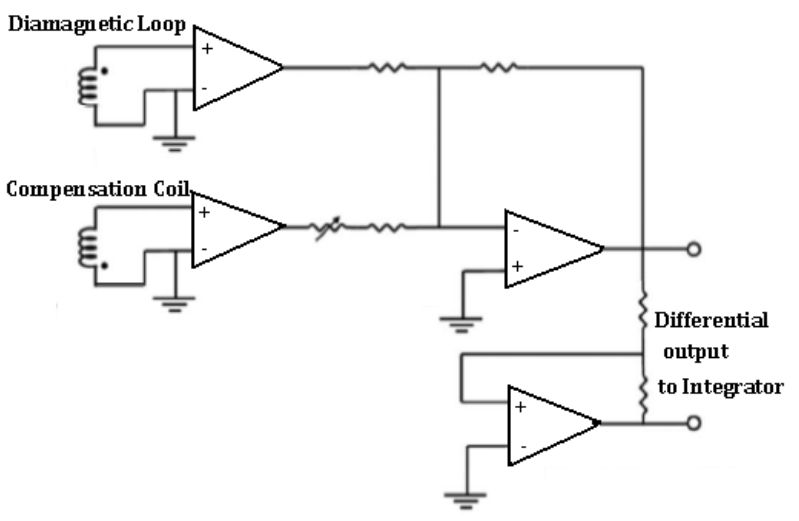

Figure 5. Electric circuit used for the diamagnetic loop and its compensation coil

According to above discussion, firstly, we measured the poloidal beta from the Eq. (9) and then the internal inductance and Shafranov shift from the Eq. (11) and Eq. (12). Results presented in the Figs. (6), (7), (8), and (9).

In the Figure (6), we plotted the plasma parameters in the absence of TF ripple. Plas ma current is observable in the Figure (6a). As shown in the Figure (6b), the measured poloidal beta is closes to one which acceptable for the 
ohmically heated tokamaks as IR-T1. A lso in the Figures (6c) and $(6 \mathrm{~d})$, the measured plasma internal inductance and Horizontal Displacement (H.D.) presented.
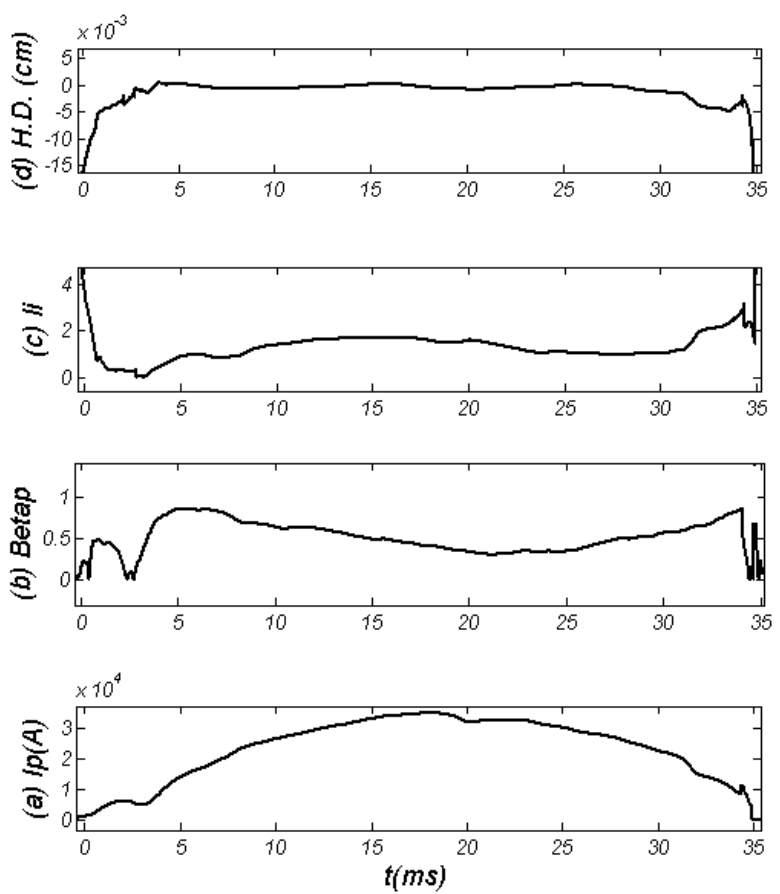

Figure 6. Plasma parameters in absence of the TF ripple, (a) Plasma current, (b) Poloidal beta, (c) Internal inductance, and (d) Horizontal Displacement (H.D.)
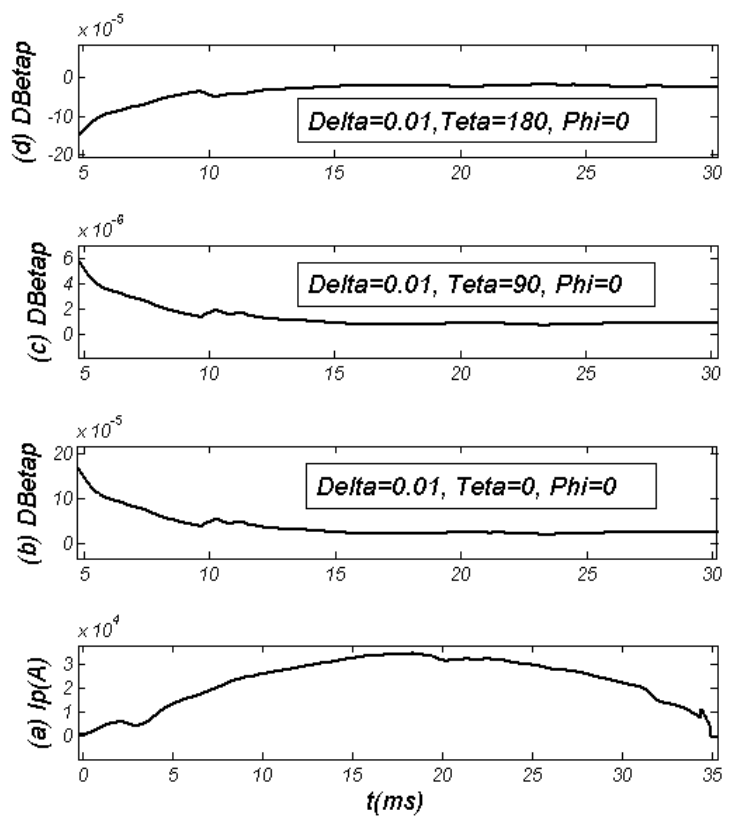

Figure 7. Effects of the equal TF ripple amplitude on the difference of poloidal beta with and without TF ripple (DBetap) at different poloidal angles

In the Figure (7), the effects of TF ripple amplitude on the difference of poloidal beta with and without TF ripple (DBetap) at different poloidal angles presented. As shown, difference between the poloidal beta in present of the TF ripple and in absent of the TF ripple is in order of the $10^{-4}$.
Also in the high field side region ( $\theta=180^{\circ}$ ) the difference is negative, whereas in low field side $\left(\theta=0^{\circ}\right)$ the difference is positive.
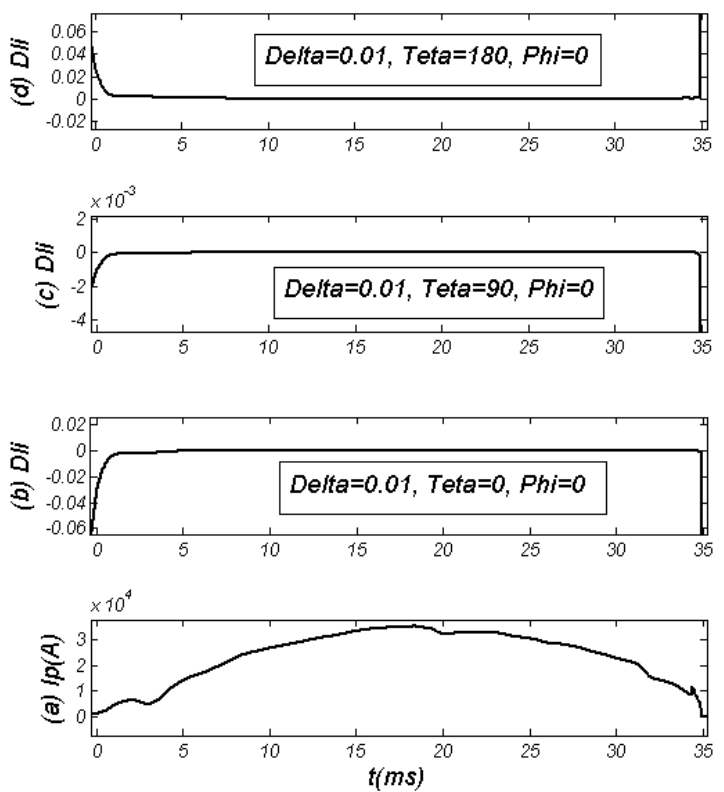

Figure 8. Effects of the equal TF ripple amplitude on the difference of internal inductance with and without TF ripple (Dli) at different poloidal angles
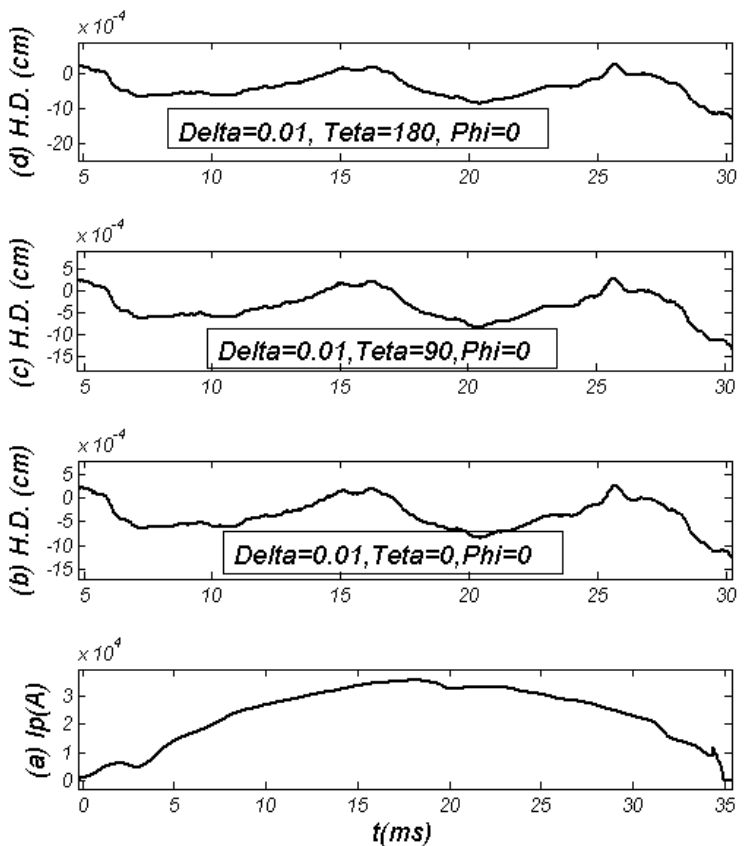

Figure 9. Effects of the equal TF ripple amplitude on the Horizontal Displacement (H.D.) at different poloidal angles

In the Figure (8), the effects of TF ripple amplitude on the difference of internal inductance with and without TF ripple (Dli) at different poloidal angles presented. As shown, difference between the internal inductance in present of the TF ripple and in absent of the TF ripple is in order of the $10^{-2}$. Also in the high field side region ( $\theta=180^{\circ}$ ), unlike the poloidal beta case, the difference is positive, 
whereas in low field side $\left(\theta=0^{\circ}\right)$ the difference is negative.

In the Figure (9), the effects of TF ripple amplitude on the Horizontal Displacement (H.D.) at different poloidal angles presented. No difference observed.

\section{Summary and Conclusions}

In this paper we presented theoretical and experimental investigation of effects of the TF ripple on the poloidal beta, internal inductance, and Shafranov shift in IR-T1 tokamak. For these purposes, array of magnetic probes and also a diamagnetic loop have been designed, constructed, and installed on outer surface of the IR-T 1. Then, poloidal and radial components of the magnetic fields and also diamagnetic flux measured. Amplitude of the TF ripple is obtained 0.01 , and also the effect of TF ripple on the poloidal beta, internal inductance, and Shafranov shift were investigated. One of the results is that difference between the poloidal beta in presence of the TF ripple and in absence of the TF ripple is in order of the $10^{-4}$, and also in the high field side region the difference is negative, whereas in the low field side the difference is positive. Another results is that difference between the internal inductance in presence of the TF ripple and in absence of the TF ripple is in order of the $10^{-2}$, and also in the high field side region unlike the poloidal beta case, the difference is positive, whereas in low field side the difference is negative. In the Shafranov shift, no difference observed. Also no sensible variation observed in the Shafranov shift due to the TF ripple on IR-T 1 toka mak plasma. The major approximations in our work are that we suppose the plasma minor radius is constant and also the TF ripple amplitude at different poloidal angles is constant, whereas the toroidal flu $\mathrm{x}$ is no constant.

\section{REFERENCES}

[1] Determination of Plasma Position using Poloidal Flux Loops and Comparison with Magnetic Probes Measurement in IR-T1 Tokamak, A. Salar Elahi et al., IEEE Trans. Plasma Science 38 (2), 181-185, (2010)

[2] A Modified Flux Loop for Determination of Plasma Position in IR-T1 Tokamak, A. Salar Elahi et al., IEEE Trans. Plasma Science 38 (9), 3163-3167, (2010)

[3] Analytical and Experimental Approach in Plasma Displacement Measurement in IR-T1 Tokamak, M. Emami, M. Ghoranneviss, A. Salar Elahi and A. Rahimi Rad, J. Plasma Phys. 76 (1), 1-8, (2009)

[4] A Novel Technique for the Measurement of Plasma Displacement in IR-T1 Tokamak, A. Salar Elahi et al., Fusion Engineering and Design 85, 724-727, (2010)

[5] Comparison between Flux Loops and Magnetic Probes in Determination of Shafranov Parameter in IR-T1 Tokamak, A.
Salar Elahi et al., Phys. Scripta 80, 045501, (2009)

[6] Two Experimental Methods for Measurement of Plasma Displacement in IR-T1 Tokamak, A. Salar Elahi et al., Phys. Scripta 80, 055502, (2009)

[7] Time Evolution of the Energy confinement Time, Internal Inductance and Effective Edge Safety Factor on IR-T1 Tokamak, A. Salar Elahi et al., Phys. Scripta 81 (5), 055501, (2010)

[8] Experimental Determination of Plasma Position Based on Two Analytical Methods in IR-T1 Tokamak, A. Salar Elahi et al., Phys. Scripta 82, 025502, (2010)

[9] The First Results of Electrode Biasing Experiments in the IR-T1 Tokamak, M. Ghoranneviss, A. Salar Elahi et al., Phys. Scripta 82 (3), 035502, (2010)

[10] Theoretical and Experimental Approach in Poloidal Beta and Internal Inductance Measurement on IR-T1 Tokamak, A. Salar Elahi et al., J. Fusion Energy 28 (4), 346-349, (2009)

[11] Effects of Resonant Helical Field (RHF) on Equilibrium Properties of IR-T1 Tokamak Plasma, A. Salar Elahi et al., J. Fusion Energy 28 (4), 416-419, (2009)

[12] Effects of Resonant Helical Field on Plasma Internal Inductance in IR-T1 Tokamak, A. Salar Elahi et al., J. Fusion Energy 28 (4), 408-411, (2009)

[13] RHF Effect on Shafranov Parameter and Shafranov Shift in IR-T1 Tokamak, A. Salar Elahi et al., J. Fusion Energy 28 (4), 412-415, (2009)

[14] Measurement of Plasma Energy Confinement Time in Presence of Resonant Helical Field in IR-T1 Tokamak, A. Salar Elahi et al., J. Fusion Energy 28 (4), 394-397, (2009)

[15] Measurement of Plasma Poloidal Beta in Presence of Resonant Helical Field in IR-T1 Tokamak, A. Salar Elahi et al., J. Fusion Energy 28 (4), 404-407, (2009)

[16] Two Semi-Empirical Methods for Determination of Shafranov Shift in IR-T1 Tokamak, A. Salar Elahi et al., J. Fusion Energy 28 (4), 390-393, (2009)

[17] Comparative Measurements of Plasma Position Using Multipole Moments Method and Analytical Solution of Grad-Shafranov Equation in IR-T1 Tokamak, A. Salar Elahi et al., J. Fusion Energy 28 (4), 385-389, (2009)

[18] Comparison between Discrete Magnetic Coils and Multipole Coils for Measurement of Plasma Displacement in IR-T1 Tokamak, A. Rahimi Rad, M. Ghoranneviss, M. Emami, and A. Salar Elahi, J. Fusion Energy 28 (4), 420-426, (2009)

[19] Plasma Magnetic Fluctuations Measurement on the Outer Surface of IR-T1 Tokamak, A. Salar Elahi et al., J. Fusion Energy 29 (1), 1-4, (2010)

[20] Investigation of Effects of Toroidal Field Ripple on Plasma Poloidal Beta in IR-T1 Tokamak, A. Salar Elahi et al., J. Fusion Energy 29 (1), 22-25, (2010)

[21] TF Ripple Effects on Plasma Energy Confinement Time in IR-T 1 Tokamak, A. Salar Elahi et al., J. Fusion Energy 29(1), 29-31, (2010)

[22] Measurement of the Shafranov Parameter in Presence of the Toroidal Field Ripple in IR-T1 Tokamak, A. Salar Elahi et al., 

J. Fusion Energy 29 (1), 26-28, (2010)

[23] Study of Effects of the Effective Edge Safety Factor on the Energy confinement Time in IR-T1 Tokamak, A. Salar Elahi et al., J. Fusion Energy 29 (1), 32-35, (2010)

[24] Experimental Study of Effects of the Internal Inductance on the Energy Confinement Time in IR-T1 Tokamak, A. Salar Elahi et al., J. Fusion Energy 29 (1), 36-40, (2010)

[25] Plasma Horizontal Displacement Measurement Using Flux Loops in IR-T1 Tokamak, A. Salar Elahi et al., J. Fusion Energy 29 (1), 62-64, (2010)

[26] Determination of the Plasma Internal Inductance and Evaluation of its Effects on Plasma Horizontal Displacement in IR-T1 Tokamak, A. Salar Elahi et al., J. Fusion Energy 29 (1), 76-82, (2010)

[27] Demonstration of Shafranov Shift by the Simplest Grad-Shafranov Equation Solution in IR-T1 Tokamak, A. Rahimi Rad, M. Emami, M. Ghoranneviss, A. Salar Elahi, J. Fusion Energy 29 (1), 73-75, (2010)

[28] Effects of Alfvenic Poloidal Flow and External Vertical Field on Plasma Position in IR-T1 Tokamak, A. Salar Elahi et al., J. Fusion Energy 29 (1), 83-87, (2010)

[29] Relations between the Plasma Diamagn etic Effect and Plasma Basic Parameters in IR-T1 Tokamak, A. Salar Elahi et al., J. Fusion Energy 29 (1), 88-93, (2010)

[30] Differences between the Toroidal and Poloidal Flux loops in the Measurement of Plasma Position in Tokamaks, A. Salar Elahi et al., J. Fusion Energy 29 (3), 209-214, (2010)

[31] Measurements of the Plasma Current Density and Q-Profiles in IR-T1 Tokamak, A. Salar Elahi et al., J. Fusion Energy 29 (3), 232-236, (2010)

[32] A Simplified Technique for the Determination of Plasma Displacement in IR-T1 Tokamak, A. Salar Elahi et al., J. Fusion Energy 29 (3), 251-255, (2010)

[33] Comparative Measurements of the Asymmetry Factor in Tokamaks using the Magnetic Probes, Poloidal and Toroidal Flux Loops, A. Salar Elahi et al., J. Fusion Energy 29 (3), 279-284, (2010)

[34] Design, Construction, and Installation of Movable Electrode Biasing System on the IR-T1 Tokamak, M. Ghoranneviss, A. Salar Elahi et al., J. Fusion Energy 29 (5), 467-470, (2010)

[35] Biasing Effect on Modifying of the Tokamak Plasma Horizontal Displacement, A. Salar Elahi et al., J. Fusion Energy 29 (5), 461-465, (2010)

[36] Measurement of the Plasma Boundary Shift and Approximation of the Magnetic Surfaces on the IR-T1 Tokamak, A. Salar Elahi et al., Brazilian J. of Physics 40 (3), 323-326, (2010)

[37] A Novel Optical Technique based on Image Processing for Determination of Tokamak Plasma Displacement, A. Salar Elahi et al., J. Fusion Energy 30 (2), 116-120, (2011)
[38] Effect of Limiter Biasing on Runaway electrons in Tokamaks, M.R. Ghanbari, M. Ghoranneviss, A. Salar Elahi et al., Phys. Scripta 83, 055501, (2011)

[39] Multipole Moments based Study on Determination of Toroidal Plasma Equilibrium Position and Shift, A. Salar Elahi, J of Fusion Energy 30 (6), 477-480, (2011)

[40] Determination of Tokamak Plasma Displacement based on Vertical Field Coil Characteristics, A. Salar Elahi et al., Fusion Engineering and Design 86, 442-445, (2011)

[41] Analytical Technique for Determination of Toroidal Plasma Displacement, A. Salar Elahi et al., J of Fusion Energy 31 (2), 191-194, (2011)

[42] Measurement of Runaway Electrons Energy by Hard X-ray Spectroscopy in a Small Circular Cross-section Tokamak, M.R. Ghanbari, M. Ghoranneviss, A. Salar Elahi and S. Mohammadi, Radiation Effects \& Defects in Solids 166 (10), 789-794, (2011)

[43] Design and Manufacturing of the Electrode Biasing System for the Tokamak, A. Salar Elahi et al., IEEE Trans. Plasma Science 40 (3), 892-897, (2012)

[44] First Results of Movable Limiter Experiments and its Effects on the Tokamak Plasma Confinement, A. Salar Elahi et al., Accepted for publication in Radiation Effects \& Defects in Solids (January 2012), DOI: 10.1080/10420150.2011.650171

[45] Investigation of Tokamak Plasma Fluctuations using Power Spectrum and FFT Analy sis of Mirnov Coils Oscillations, Z. Goodarzi, M. Ghoranneviss and A. Salar Elahi, Accepted for the publication in J Fusion Energy (March 2012), DOI: 10.1007/s 10894-012-9526-4

[46] Controlling the Energy of Runaway Electrons by Emissive Limiter Biasing in Tokamaks, M.R. Ghanbari, M. Ghoranneviss, A. Salar Elahi et al., Phys. Scripta 85 (5), 055502, (2012)

[47] Laser Fusion Energy from $p-{ }^{7} \mathrm{Li}$ with Minimized Radioactivity, M. Ghoranneviss, A. Salar Elahi, H. Hora, G.H. Miley et al., Accepted for the publication in Laser and Particle Beams (May 2012), DOI: 10.1017/S0263034612000341

[48] Design and Fabrication of Emissive Biased Limiter and its Effect on Tokamak Plasma, M. Ghoranneviss et al., Accepted for the publication in Radiation Effects and Defects in Solids (June 2012)

[49] New Approaches on Application of Multipole Moments for Determination of Toroidal Plasma Shift, A. Salar Elahi et al., Accepted for the publication in Radiation Effects and Defects in Solids (June 2012), DOI: 10.1080/10420150.2012.706609

[50] Design and Construction of Hot Limiter Biasing System for the Tokamak, A. Salar Elahi et al., Accepted for the publication in Radiation Effects and Defects in Solids (June 2012), DOI: $10.1080 / 10420150.2012 .706607$

[51] Estimating of Time dependence of Edge Plasma Turbulence in IR-T1 Tokamak, K. Mikaili, M. Ghoranneviss, A. Salar Elahi et al., Accepted for the publication in J Fusion Energy (July 2012), DOI: 10.1007/s10894-012-9563-Z 